\title{
Energy Efficient Resource Allocation using Improved BSP Technique in Cloud Environment
}

\author{
M.J.Abinash, V. Vasudevan
}

\begin{abstract}
Cloud computing allows the user to access their resources from data center. The energy consumed by each resources will leads to low performance in the data center. The existing work deals with heuristic resource allocation which concentrates only on bottleneck resources. This research work proposes BSP algorithm to allocate the data center resources efficiently in terms of dynamic information and energy consumption. BSP is a placement technique which provides high quality placement for resources based on optimization process. The agent provide authentication to each user to ensure the privacy of the system.

The overall resource allocation is achieved by BPS technique by performing continuous deployment and ongoing optimization. Continuously deployment is efficiently allocating the resources based on demand risk score. If any virtual machine in the cloud environment is overloaded, the ongoing optimization technique is applied to migrate the resources from overloaded VM to idle VM. The benefit of our improved BSP technique with the comparison of the results of the existing system is shown in performance analysis.
\end{abstract}

Index Terms-Cloud data center, Backward Speculative Placement (BSP), resource allocation, resource prediction.

\section{INTRODUCTION}

The active provisioning of computing services usually make use of Virtual Machine (VM) for integration and separating the atmosphere purposes is classified as a new exemplar in cloud computing. Cloud infrastructure should fulfill the characteristics which are essential to support the services. Consumption based metering measures are managed by the tools. . This faces complex management and underutilization of resources. The resource pools facilitate centralized management of resources and enables faster resource provisioning

Cloud benefits offer significantly contributed to corporate companies by mitigating them. It will focus the importance of installing the hardware and software infrastructures. To understand the power of Cloud computing fully, meet various consumer requirements the service providers have to be stretchy in their delivery, while keeping the consumers inaccessible from the underlying infrastructure. A data center deployment is concerned with high performance, and without paying attention to energy consumption this demand has been fulfilled. By understanding the existing physical infrastructure the elements and processes will begins.
As energy cost increases, energy efficiency is optimized by shifting the data center resource managing for purity, to maintain high performance. Therefore, Due to high energy costs profit margin is not reduced.

These measures are adopted to ensure by the cloud service providers. The increase in the cost of the energy highly possible hazard as it boosting the Total Cost of Ownership (TCO) and minimizes the Return on Investment (ROI) of the infrastructure Cloud. Nowadays, the highly possible computing service providers have formed a world group is called as the eco friendly Grid to increase energy efficiency for data centers and reduction of the environmental impact. Minimization of energy consumption of Cloud infrastructures should be reduced by the providers, while ensuring the service deliverance.

The issue to lower the energy use of group of data centers is difficult and multifaceted because of mathematical applications and data that are rising immediately will increase wide server range and disks which are required to process them speed enough within the required time period. Eco friendly Cloud computing is predicted not only to attain the high processing and usage of a computing infrastructure, but also to reduce the consumption of energy. Energyefficient manner is managed by green cloud computing to drive and deal with this problem. Cloud resources nor only satisfies the Quality of Service but also reduces energy usage.

\section{PROBLEM DEFINITION}

Data centers in cloud environment allocate the user's tasks to the virtual machines. Network traffic and storage facilities are main issues in placement of VM in cloud environment. Due to high network traffic, more amount of energy is consumed for resource allocation, which is the main cause of poor performance of the system. To solve this problem, a new solution is prepared that allows reducing the energy consumption while allocating the resources. During the resource allocation tasks are allocated to the available resource without loss of energy.

Revised Manuscript Received on September 15, 2019.

M.J.Abinash, Research Scholar, Department of Information Technology, Kalasalingam Academy of Research and Education (Deemed to be University), Krishankoil, Tamilnadu. mj.abinash@gmail.com

V. Vasudevan, Senior Professor, Department of Information Technology, Kalasalingam Academy of Research and Education (Deemed to be University), Krishankoil, Tamilnadu. vasudevan_klu@ yahoo.co.in 


\section{DATA FLOW DIAGRAM}

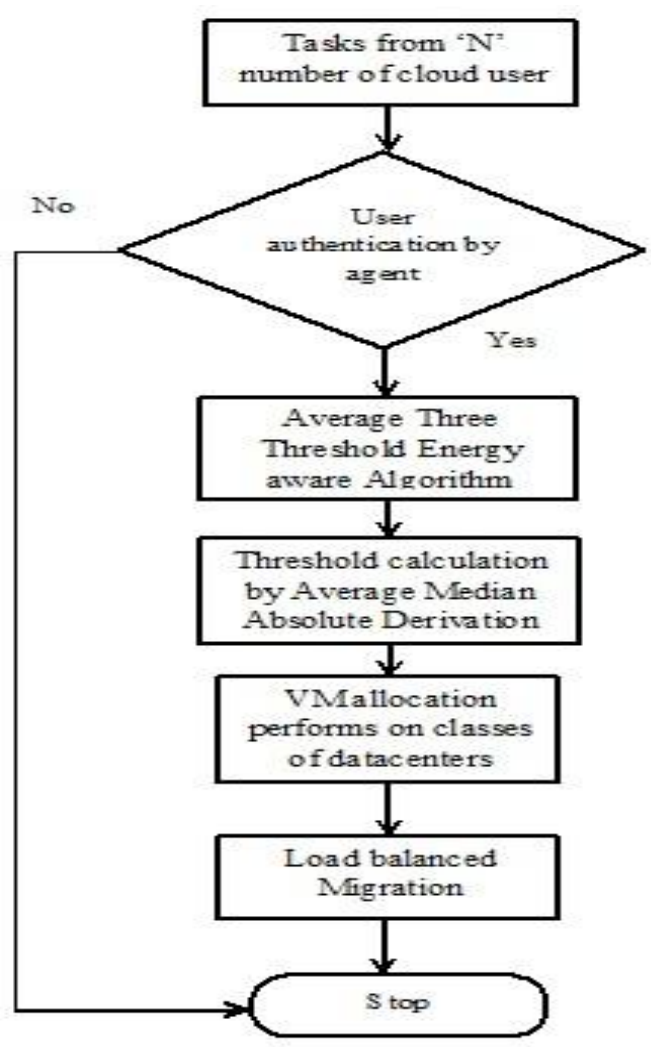

Fig. 1. Flowchart

\subsection{System Architecture}

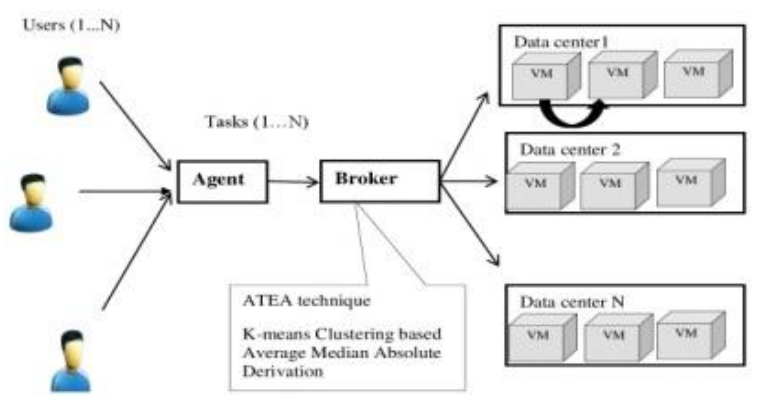

Fig. 2.System Architecture

\section{METHODOLOGY}

\subsection{Creation of Cloud}

To provide efficient resource allocation, the cloud environment is comprised with users, agent, broker, and data center. The multiple numbers of user tasks are received by the agent. Then the received tasks are transformed to brokers to achieve optimal resource allocation. The broker applies the Backward Speculative Placement (BPS) technique to provide placement to virtual machine and also responsible for migrate the tasks from overloaded virtual machine to idle virtual machine.

\subsection{Agent based Authentication}

In our proposed method, the agent is responsible to provide authentication to each user who are involving to allocate their tasks to resources. Our proposed system allows only the legitimate users who are authenticated by agent. Then the broker receives the tasks from the authenticated users to provide optimal placement.

\subsection{VM Placement}

In our proposed method, the VM placement is efficiently achieved by Broker by applying BPS technique. We have proposed BPS technique based Continuous deployment to allocate the resource efficiently based on the demand risk. The demand risk score is calculated to measure the demand dissatisfaction of a host in a last time instants. Based on the demand risk score the tasks are allocated to virtual machines.

\subsection{VM Migration}

In our proposed system, the migration process is achieved by BPS based ongoing optimization technique. It improves the efficiency of the system by migrating the tasks. If the virtual machine is overloaded by tasks, the ongoing optimization is performed to migrate the tasks to idle virtual machines. Then the energy consumption is minimized by shut down the idle physical machines in the host.

\subsection{Performance Assessment}

The main objective of the performance assessment is analyzing different load condition under BSP's quality and performance. The performance of the proposed system is evaluated by using following metrics as execution time and demand satisfaction which provides better results compared to existing system.

\section{PROPOSED ALGORITHM}

\section{BSP based Resource Allocation}

This paper proposed Backward Speculative Placement (BSP) technique for efficient resource allocation. BSP is a placement technique which provides high quality placement for resources based on optimization process. We propose BSP to overcome the traditional scheduling problems. There are two phases in BSP technique such as, Continuous deployment and ongoing optimization. Continuous deployment is used allocate the tasks to available resources. Ongoing optimization is mainly focused to migrate the virtual machine from one physical machine to another physical machine.

\section{Continuous Deployment}

Cloud computing system continuously received number of tasks from the users. This type of actions demands the immediate assignment of tasks to the available resources. We proposed continuous deployment to assign the resource efficiently based on required risk score. The main purpose of demand risk is to measure the demand dissatisfaction of a host in last time instants. Based on the demand risk score the tasks are allocated to the virtual machine in the concerned data center.

\section{Ongoing optimization}

Ongoing optimization procedure is performed to improve the efficiency of the system by migration process. In this process, the overloaded virtual machines are identified for migration process. Then the overloaded virtual machines are migrated to underutilized host. The idle physical machines in the host are shut down to minimize the energy consumption.

\section{RESULT}

Our proposed algorithm consists of continuous deployment and ongoing optimization process. Demand risks are calculated in continuous deployment. The overall 
resource utilization on cloud is shown in Fig.3.

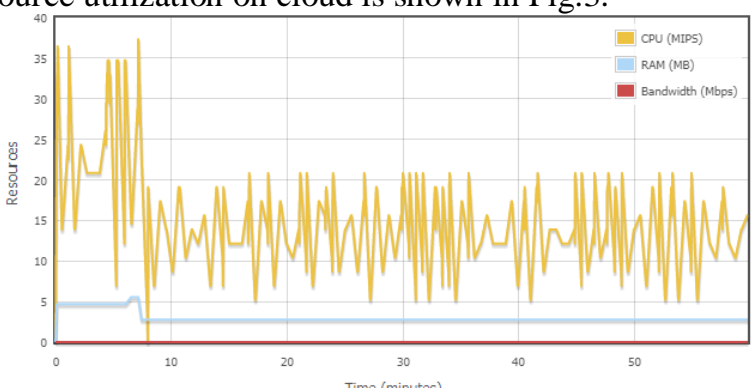

Fig.3 Resource Utilization

The overall Power consumption on cloud is shown in Fig.4 .

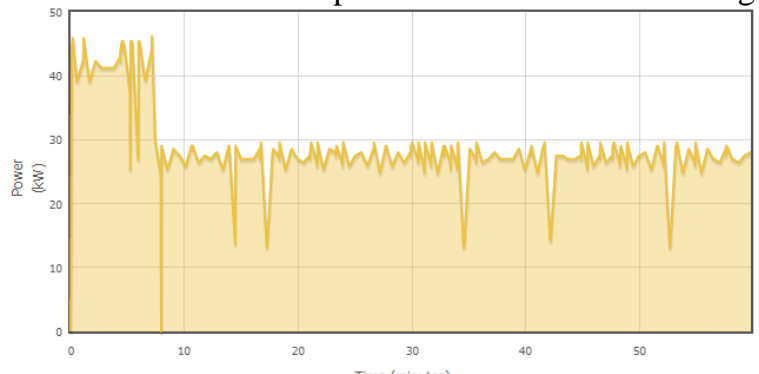

Fig.4 Power Consumption

Number of migration processes for every task performed is shown in Fig. 5.

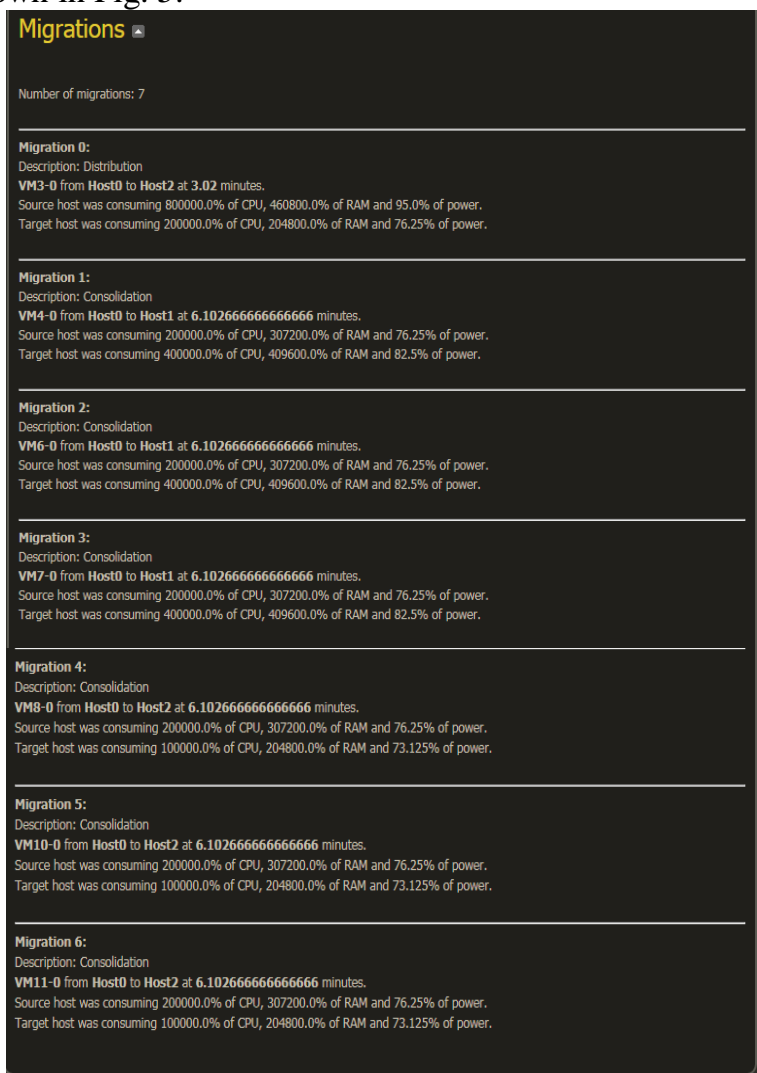

Fig.5 VM Migration

Comparison graph between BSP and CPLEX algorithm is shown in Fig.5.

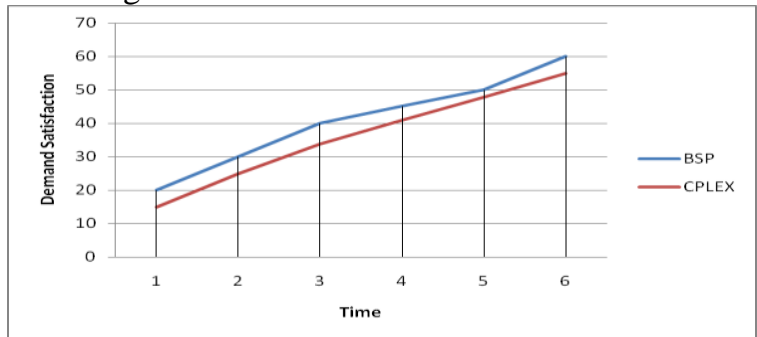

Fig. 6 Demand Satisfaction

\section{CONCLUSION}

We have proposed the energy efficient resource allocation method by Backward Speculative Placement algorithm. By using efficient host based on demand risk score. Then the overloaded virtual machines are migrated to under utilized host by performing ongoing optimization technique based on BPS algorithm. The privacy of the system is enhanced by agent that provide authentication for each user. The energy efficiency of the system is minimized by turn off all the idle hosts. The performance of our proposed system is analysed based on demand satisfaction and execution time which provides better results compared to existing system. In future, we have planned to improve the proposed system in the area of scheduling by applying meta heuristic algorithm.

\section{REFERENCES}

[1] N. Bobroff, A. Kochut, and K. Beaty (2007). Dynamic placement of virtual machines for managing sla violations. In Integrated Network Management, '07. IM '07. 10th IFIP/IEEE InternationalSymposium on, pages $119-128$

[2] D. Breitgand and A. Epstein(2011). Sla-aware placement of mult virtual machine elastic services in compute clouds. In Integrated Network Management (IM), '11 IFIP/IEEE International Symposium on, pages $161-168$

[3] Ming Chen, Hui Zhang, Ya-Yunn Su, Xiaorui Wang, Guofei Jiang, and Kenji Yoshihira(2011). Effective vm sizing in virtualized data centers. In Integrated Network Management, IM'11, pages 594-601.

[4] Christopher Clark, Keir Fraser, Steven Hand, Jacob Gorm Hansen, Eric Jul, Christian Limpach, Ian Pratt, and Andrew Warfield (2005). Live migration of virtual machines. In Proceedings of the 2nd conference on Symposium on Networked Systems Design \& Implementation, NSDI'05, pages 273-286, Berkeley, CA, USA. USENIX.

[5] Zhenhuan Gong and Xiaohui Gu (2010). Pac: Pattern-driven application consolidation for efficient cloud computing. In Modeling, Analysis Simulation of Computer and Telecommunication Systems (MASCOTS), 2010 IEEE International Symposium on, pages 24-33

[6] D. Jayasinghe, C. Pu, T. Eilam, M. Steinder, I. Whalley, and E. Snible (2011). Improving performance and availability of services hosted on iaas clouds with structural constraint-aware virtual machine placement In Services Computing (SCC), 2011,IEEE International Conference on, pages 72-79

[7] M. Korupolu, A. Singh, and B. Bamba (2009). Coupled placement in modern data centers. In Parallel Distributed Processing, '09. IPDPS '09. International Symposium on, pages 1-12.

[8] Wubin Li, Johan Tordsson, and Erik Elmroth(2011). Virtual machine placement for predictable and time-constrained peak load. In In Proceedings of the 8th International Workshop on Economics of Grids, Clouds, Systems, and Services, LNCS. Springer-Verlag.

[9] Xiaoqiao Meng, Canturk Isci, Jeffrey Kephart, Li Zhang, Eric Bouillet, and Dimitrios Pendarakis (2010). Efficient resource provisioning in compute clouds via vm multiplexing. In Proceeding of the 7 th international conference on Autonomic computing, pages $11-20$, New York, NY, USA.

[10] Xiaoqiao Meng, V. Pappas, and Li Zhang (2010). Improving the scalability of data center networks with traffic-aware virtual machine placement. In INFOCOM, '10 Proceedings IEEE, pages 1-9.

[11] Saranya.S, Murugan.B.S (2014). Intelligent Scheduling System for Dynamic Resource Allocation in Cloud Computing. In International Journal of Advanced Research in Computer Science \& Technology (IJARCST 2014) IJARCST All Rights Reserved 284 Vol. 2 Issue Special 1 Jan-March 